\title{
Calculs et mesures de l'activation induite dans les bétons de protection d'un accélérateur d'ions de haute énergie
}

\author{
L. MASSON, L. BOURGOIS*
}

(Manuscrit reçu le ler février 2000, révisé le 29 mars 2000, accepté le ler mai 2000)

RÉSUMÉ Le synchrotron Saturne II, installé sur le site du CEA Saclay fonctionnant depuis 1979 devrait être démantelé dans les prochaines années. Saturne pouvait accélérer des protons, des deutons, des hélions, et des ions lourds (jusqu'au Krypton) avec une énergie en protons de 100 à $2900 \mathrm{MeV}$. Des calculs avec un code Monte Carło (FLUKA), couplé à un code de calcul d'activation/évolution (DARWIN/PEPIN) ont été menés pour prédire l'activation dans les protections en béton de l'accélérateur. Les produits d'activation sont en majorité dus à des réactions de capture de type $(n, \gamma), 22 \%$ de l'activation étant due à des neutrons d'énergie supérieure à $20 \mathrm{MeV}$-type (n,p); (n, spallation) etc. Ces calculs ont été comparés à des mesures des émetteurs $\gamma$, du ${ }^{3} \mathbf{H}$, du ${ }^{45} \mathrm{Ca}$ et du ${ }^{55} \mathrm{Fe}$ d'échantillons prélevés par carottage sur des bétons ordinaires $\left(2,35 \mathrm{~g} \mathrm{~cm}^{-3}\right)$, lourd baryté $\left(3,6 \mathrm{~g} \mathrm{~cm}^{-3}\right)$ et très lourd riblonné $\left(4,8 \mathrm{~g} \mathrm{~cm}^{-3}\right)$. Le rapport entre calcul et mesure varie de 1 à 6 suivant le type de béton considéré et la profondeur de protection. De même, les mesures ont été comparées à d'autres pour des accélérateurs différents (énergie et particules). On montre que l'activation dépend de la charge intégrée sur les cibles de production et de l'énergie des particules notamment pour le profil d'activation en profondeur.

ABSTRACT Calculations and measurements of activation in concrete radiological shielding produced by a high energy ions accelerator.

Saturne II synchrotron is located on CEA Saclay site and was running since 1979. It should be dismantled in the next few years. Saturne accelerated protons, deuterons, helions and heavy charged particles (up to Krypton) with protons energie ranged from 100 to $2900 \mathrm{MeV}$. Monte Carlo calculations (FLUKA) coupled with activation/evolution computer codes (DARWIN/PEPIN) were used in order to predict activation in concrete radiological shielding of the accelerator. Activation products are mainly due to $(n, \gamma)$ capture reaction, $22 \%$ of the activation comes from neutrons with energy higher than $20 \mathrm{MeV}$ - (n,p); (n, spallation) etc. Those calculations were compared to $\gamma$ rays $,{ }^{3} \mathrm{H},{ }^{45} \mathrm{Ca}$ and ${ }^{55} \mathrm{Fe}$ measurements on samples taken from different concrete shieldings: ordinary $\left(2,35 \mathrm{~g} \mathrm{~cm}^{-3}\right)$, heavy with Barium $\left(3,6 \mathrm{~g} \mathrm{~cm}^{-3}\right)$, extra heavy with iron $\left(4,8 \mathrm{~g} \mathrm{~cm}^{-3}\right)$. The ratio between calculation and measurement varies from 1 to 6 depending on the kind of concrete and the protection depth. Besides, measurements were compared to those obtained by other 
accelerators (differents energies and particles). It shows up that activation depends on integrated charge over production targets and on particles energy, especially on in-depth activation profile.

\section{Introduction}

Le synchrotron Saturne II, installé sur le site du CEA Saclay fonctionnant depuis 1979 a été arrêté en décembre 1997. Il devrait être démantelé dans les prochaines années. L'accélérateur et ses bâtiments annexes occupent une surface de plus de 4 hectares. Saturne pouvait accélérer des protons, des deutons, des hélions, et des ions lourds (jusqu'au Krypton) avec une énergie en protons de 100 à $2900 \mathrm{MeV}$ (I.R.F., 1987). La figure 1 donne la configuration de l'accélérateur en 1998.

La connaissance de l'activation des protections en béton est très importante en particulier pour leur caractérisation radiologique en cas d'évacuation ou d'élimination. En effet, les protections radiologiques de Saturne sont constituées de 22500 blocs de béton amovibles soit près de 34000 tonnes (Masson, 1999a).

Cet article a pour but de comparer l'activation en profondeur dans les protections radiologiques en béton, calculée par un code Monte-Carlo couplé à un code de calcul d'activation et des mesures effectuées par carottage. De même, les résultats seront comparés à d'autres sur différents accélérateurs de types différents.

\section{Calculs de l'activation des bétons}

\subsection{Matériel et méthodes}

Afin de déterminer la zone d'activation maximale, L. Masson (1999a) a établi un historique de l'installation. Celui-ci, a consisté à :

- déterminer les points de pertes (localisation, géométrie);

- donner les conditions de fonctionnement de l'accélérateur (type de particules, énergie, intensité).

Cet historique montre que c'est au niveau du septum de l'aimant de l'extraction 2 (SD2) que l'on observe le maximum de perte. Celui-ci peut être modélisé par une cible de cuivre de $20 \mathrm{~cm}$ de long et $8 \mathrm{~cm}$ de diamètre.

Le tableau I donne les particules perdues dans SD2 pendant les 19 ans d'accélération. Les pertes seront modélisées par un faisceau de protons de $2 \mathrm{GeV}$ d'intensité $1 \times 10^{10} \mathrm{p} \mathrm{s}^{-1}$ avec un cycle de fonctionnement de 6 mois par an (fréquence d'utilisation de la machine). 
ACTIVATION INDUITE DANS UN ACCÉLÉRATEUR D'IONS DE HAUTE ÉNERGIE

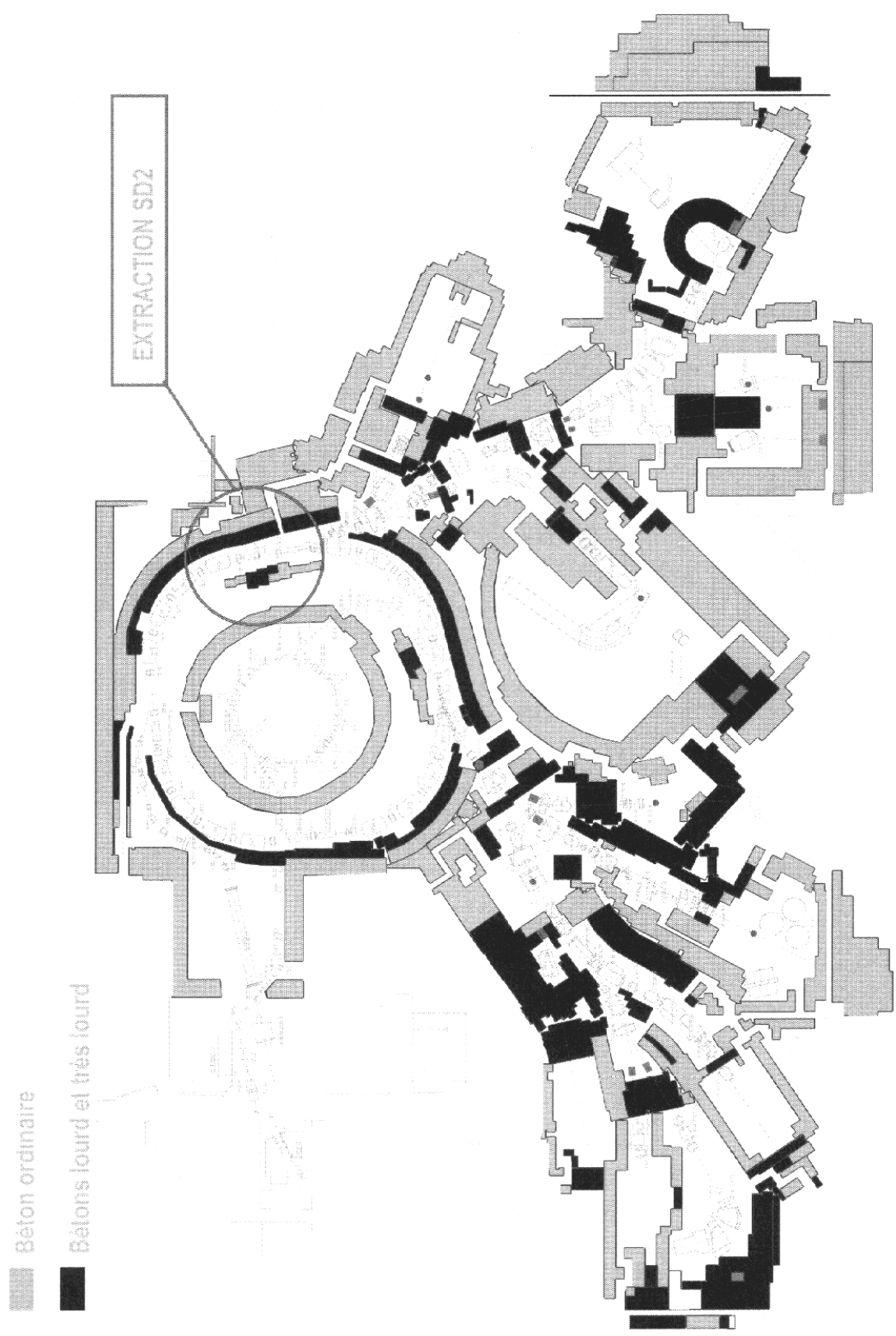

Fig. 1. - Plan de Saturne 2.

Saturne 2 plan view. 
TABLEAU I

Répartition du nombre de particules perdues en SD2.

Beam losses repartition in SD2.

\begin{tabular}{|lccccccccc|}
\hline & \multicolumn{2}{c}{$\mathrm{E}<0,5 \mathrm{GeV}$} & \multicolumn{2}{c}{$0,5<\mathrm{E}<1 \mathrm{GeV}$} & \multicolumn{2}{c|}{$\mathrm{E}>1 \mathrm{GeV}$} & \multicolumn{2}{c|}{ Total } \\
Particules & Nombre & $\%$ & \multicolumn{2}{c}{ Nombre } & $\%$ & Nombre & $\%$ & Nombre & $\%$ \\
\hline proton & $8,1 \times 10^{17}$ & 29,1 & $1,2 \times 10^{18}$ & 41,5 & $8,2 \times 10^{17}$ & 29,4 & $2,8 \times 10^{18}$ & 64,6 \\
deuton & $4,4 \times 10^{17}$ & 46,8 & $3,8 \times 10^{17}$ & 39,9 & $1,3 \times 10^{17}$ & 13,3 & $9,4 \times 10^{17}$ & 21,9 \\
hélium-3 & $1,6 \times 10^{15}$ & 1,8 & $6,0 \times 10^{16}$ & 68,2 & $2,6 \times 10^{16}$ & 30,0 & $8,8 \times 10^{16}$ & 2,0 \\
\hline hélium-4 & $4,4 \times 10^{17}$ & 88,8 & $4,7 \times 10^{16}$ & 9,5 & $8,6 \times 10^{15}$ & 1,7 & $5,0 \times 10^{17}$ & 11,5 \\
\hline ions lourds & - & - & $3,4 \times 10^{12}$ & 0,6 & $5,2 \times 10^{14}$ & 99,4 & $5,2 \times 10^{14}$ & $<0,1$ \\
\hline Total & $1,7 \times 10^{18}$ & 39,3 & $1,6 \times 10^{18}$ & 38,1 & $9,8 \times 10^{17}$ & 22,7 & $4,3 \times 10^{18}$ & 100,0 \\
\hline
\end{tabular}

TABLEAU II

Composition chimique des bétons de l'accélérateur Saturne $\left(\mu \mathrm{g} \mathrm{g}^{-1}\right)$.

Constituent elements $\left(\mu \mathrm{g} \mathrm{g}^{-1}\right)$ in different kinds of concrete.

\begin{tabular}{|c|c|c|c|c|c|c|c|c|c|}
\hline \multirow[b]{2}{*}{ Elément } & \multicolumn{2}{|c|}{$\begin{array}{c}\text { Béton } \\
\text { ordinaire }\end{array}$} & \multicolumn{2}{|c|}{$\begin{array}{l}\text { Béton } \\
\text { très lourd }\end{array}$} & \multirow[b]{2}{*}{ Élément } & \multicolumn{2}{|c|}{$\begin{array}{c}\text { Béton } \\
\text { ordinaire }\end{array}$} & \multicolumn{2}{|c|}{$\begin{array}{l}\text { Béton } \\
\text { très lourd }\end{array}$} \\
\hline & m & $\sigma$ & m & $\sigma$ & & m & $\sigma$ & m & $\sigma$ \\
\hline $\mathrm{Si}$ & 243200 & 50100 & 18870 & 10700 & $\mathbf{R b}$ & 37.29 & 18,12 & & \\
\hline Al & 12400 & 460 & 294 & 114 & $\mathbf{Z r}$ & 49,2 & 19,1 & & \\
\hline $\mathrm{Fe}$ & 6600 & 1000 & 522400 & 159800 & Nb & 1,45 & 0,22 & & \\
\hline Mn & 23 & 9 & 17 & 11 & $\mathbf{R u}$ & 1,77 & 0,66 & & \\
\hline $\mathrm{Mg}$ & 360 & 70 & 103 & 56 & Cd & 0,16 & 0,04 & & \\
\hline $\mathrm{Ca}$ & 177100 & 46800 & 52500 & 21900 & In & 0,03 & 0,02 & & \\
\hline $\mathrm{Na}$ & 200 & 100 & 16 & 8 & Sn & 1,54 & 0,86 & & \\
\hline K & 6800 & 2800 & 53 & 36 & $\mathrm{Sb}$ & 0,45 & 0,16 & 22 & 7,48 \\
\hline $\mathrm{Ti}$ & 33 & 15 & 31 & 8 & Cs & 2,1 & 0,8 & & \\
\hline P & 95 & 45 & 33 & 25 & La & 6,63 & 1,10 & 6,17 & 2,80 \\
\hline C & 39500 & 15100 & 690 & 320 & $\mathrm{Ce}$ & 15,38 & 5,88 & 7,24 & 2,60 \\
\hline H & 66 & 9 & 27 & 13 & Pr & 1,99 & 0,61 & & \\
\hline S & 170 & 10 & 28700 & 19500 & Nd & 7,23 & 3,19 & & \\
\hline O & 480800 & 18900 & 147600 & 51800 & Eu & 0,41 & 0,18 & 0,36 & 0,22 \\
\hline Ba & 52 & 40 & 97300 & 70700 & Gd & 1,49 & 0,68 & & \\
\hline $\mathrm{Sr}$ & 28 & 4 & 340 & 240 & Sm & 1,54 & 0,63 & 1,9 & 0,5 \\
\hline $\mathrm{Be}$ & 0,92 & 0,29 & & & Tb & 0,25 & 0,11 & 0,36 & 0.12 \\
\hline $\mathrm{Cl}$ & 255 & 65 & & & Dy & 1,37 & 0,68 & -4 & \\
\hline $\mathrm{Sc}$ & 2,06 & 1,27 & 0,99 & 0,41 & Но & 0,29 & 0,16 & & \\
\hline $\mathrm{Cr}$ & 31,19 & 9,76 & 128,25 & 69,90 & Er & 0,71 & 0,37 & & \\
\hline V & 17 & 3 & & & Tm & 0,11 & 0,06 & & \\
\hline Co & 2,91 & 0,45 & 61,75 & 24,28 & $\mathrm{Yb}$ & 0,78 & 0,41 & 0,66 & 0,29 \\
\hline $\mathrm{Ni}$ & 21,8 & 2,9 & & & $\mathrm{Lu}$ & 0,12 & 0,06 & 0,10 & 0,03 \\
\hline $\mathrm{Cu}$ & 7,4 & 2,1 & & & Hf & 1,09 & 0,44 & 0,31 & 0,06 \\
\hline $\mathrm{Zn}$ & & & 88 & 45 & $\mathrm{Ta}$ & 0,18 & 0,04 & 0,20 & 0,02 \\
\hline Ga & 2,9 & 0,8 & & 5 & W & 0,71 & 0,42 & 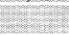 & 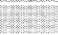 \\
\hline $\mathrm{Ge}$ & 0,60 & 0,17 & 5 & 2 & $\mathbf{P b}$ & 17,75 & 2,86 & & \\
\hline As & 5,41 & 0,81 & & $x=$ & Th & 2,18 & 0,68 & 0,95 & 0,47 \\
\hline $\mathrm{Br}$ & 1,2 & 0,3 & & & U & 1,03 & 0,14 & & \\
\hline Y & 8,82 & 3,41 & & & & & & & \\
\hline
\end{tabular}


On a effectué un inventaire des blocs de protection radiologique autour de ce point de pertes (Masson, 1999a). On a recensé trois types de béton :

- ordinaire de densité 2,35 ;

- lourd baryté de densité 3,6;

- très lourd riblonné de densité 4,8 .

La détermination des compositions chimiques en éléments majeurs et traces dans les bétons, a été effectuée par le laboratoire Pierre Süe du CEA Saclay (Gaudry et al., 1997) avec des méthodes de radioactivation neutronique ou de spectrométrie de masse (ICPMS). La composition des bétons figure dans le tableau II.

Avec ces données, Klein et Diop (1999) ont effectué les calculs d'activation dans la zone SD2. Le code FLUKA version 94/3 (Fasso et al., 1994) a été utilisé pour le transport des particules chargées et neutres. La géométrie de calcul est donnée dans la figure 2. Ce code a été couplé au système DARWIN/PEPIN2 développé au CEA (Tsilanizara et al., 1999) qui permet de résoudre de manière analytique ou numérique les équations différentielles couplées régissant les filiations radioactives en particulier, celles relatives aux produits d'activation et de spallation. Pour calculer l'activité d'un matériau dans une région donnée, il faut résoudre l'équation régissant le nombre de noyaux $\mathrm{N}_{i}(t)$ d'un isotope $i$ quelconque en fonction du temps. Cette équation s'écrit :

$$
\frac{\mathrm{d} N_{i}(t)}{\mathrm{d} t}=\Sigma \gamma_{s(j \rightarrow i)} N_{j}(t)+\Sigma_{j} \lambda_{j} b_{(j \rightarrow i)} N_{j}(t)-\lambda_{i} N_{i}(t)+\Sigma\left[\int_{E} \sigma_{n, j}(E) \psi_{n}(E) \mathrm{d} E\right] N_{j}(t)
$$

avec $\omega_{s(j i)}$ : production par spallation $(\mathrm{E}>20 \mathrm{MeV})$ fournis par FLUKA, $<_{j} b_{(j i)}$ : alimentation par décroissance de noyaux pères de $j$ vers $i$, $-<_{i}$ : disparition par décroissance radioactive de l'isotope $i$, $-{ }_{n j} \infty_{n}$ : production par réactions neutroniques $(\mathrm{E}<20 \mathrm{MeV})$ sur $j$.

L'enchaînement des différents codes de calcul est donné dans la figure 3.

\subsection{Résultats des calculs}

Les résultats de calculs de l'activité massique en fonction de la profondeur dans le bloc de béton sont donnés dans la figure 4 pour les bétons ordinaires et dans la figure 5 pour les bétons très lourds. Ces résultats sont donnés pour un an de décroissance après le dernier fonctionnement de la machine. L'activité totale tient compte de la radioactivité naturelle de ces bétons $\left({ }^{40} \mathrm{~K},{ }^{87} \mathrm{Rb},{ }^{234} \mathrm{Th},{ }^{234 \mathrm{~m}} \mathrm{~Pa},{ }^{234} \mathrm{U},{ }^{238} \mathrm{U}\right)$ soit $0,2 \mathrm{~Bq} \mathrm{~g}^{-1}$ pour le béton ordinaire et $0,07 \mathrm{~Bq} \mathrm{~g}^{-1}$ pour le béton très lourd.

\subsection{Discussion}

L'activation est due en grande partie aux réactions de capture (n, $\gamma)$ dont la section efficace est maximale pour les neutrons thermiques. C'est le cas des réactions 


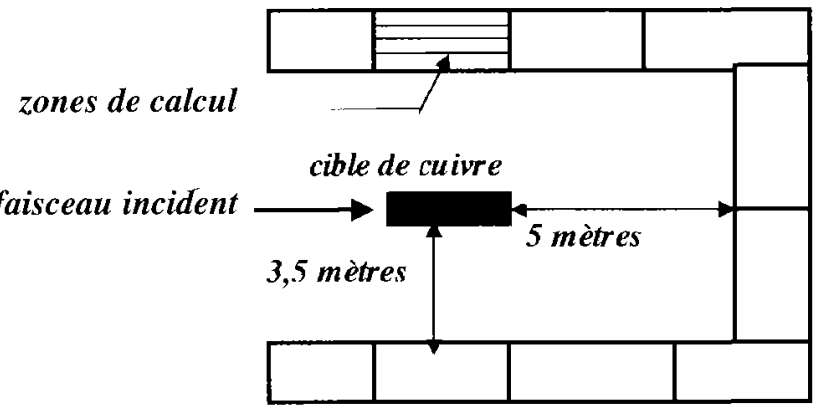

\section{blocs}

de béton

Fig. 2. - Géométrie de calcul.

Calculation geometry model.

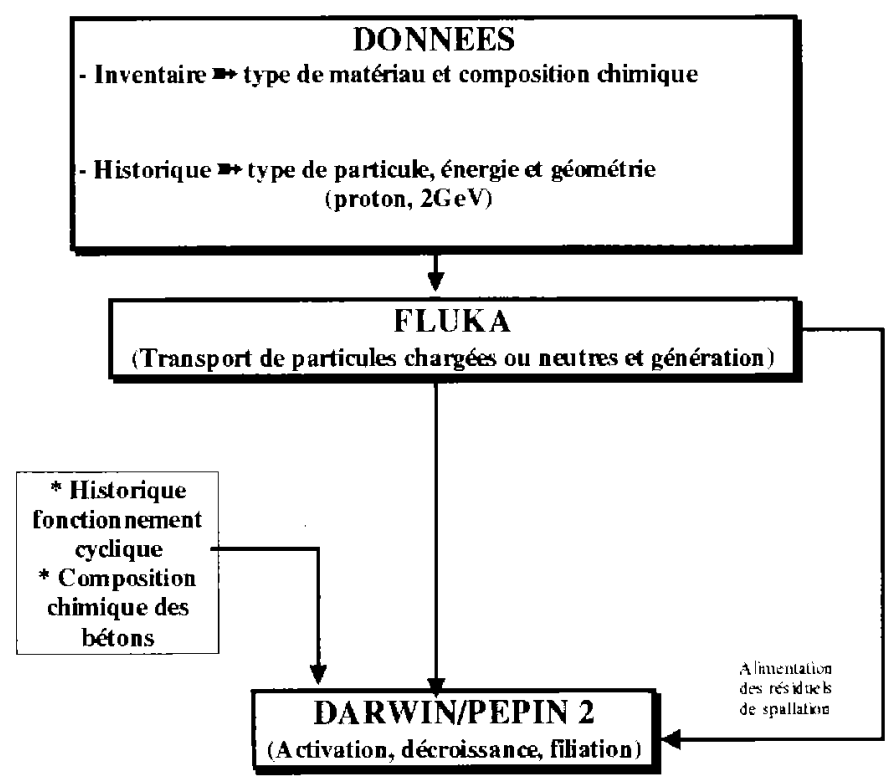

Fig. 3. - Enchaînement des codes de calcul.

Computer codes calculation sequences. 


\section{Activité en Bq.g-1}

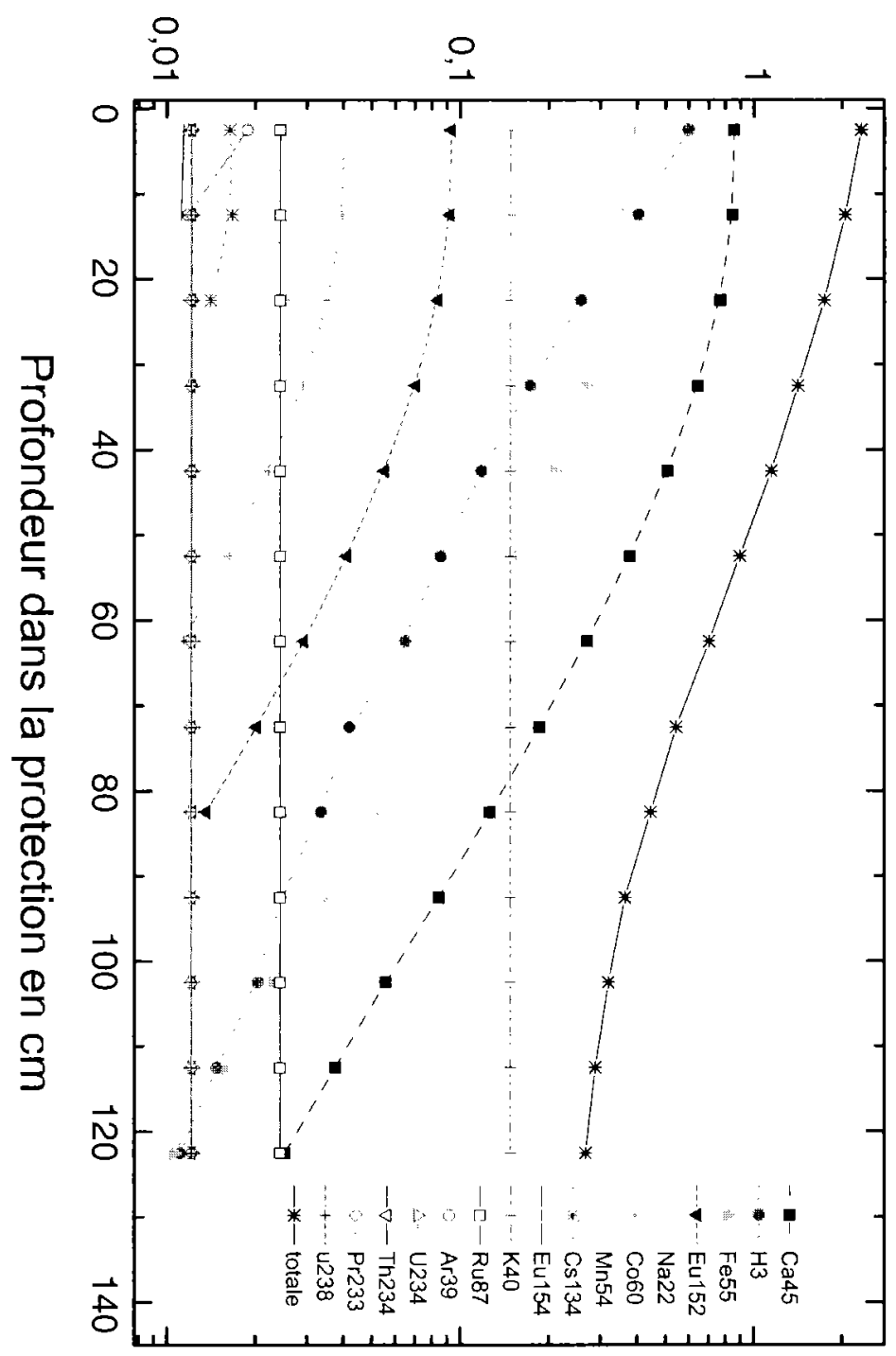

Fig. 4. - Variation de l'activité massique calculée en fonction de la profondeur pour un béton ordinaire.

Calculated in-depth specific activity profiles in ordinary concrete. 


\section{Activité en Bq. $\mathrm{g}^{-1}$}

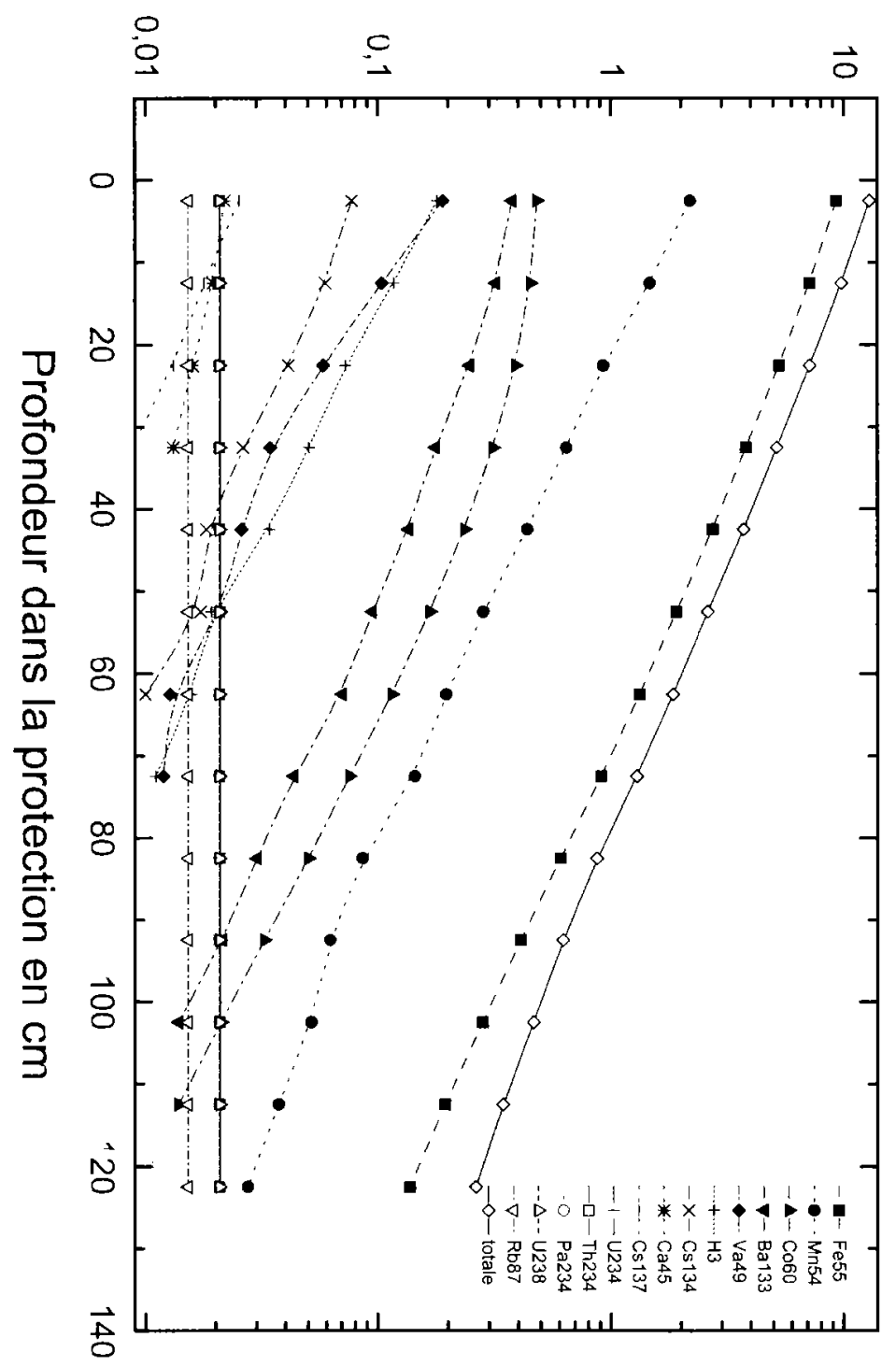

Fig. 5. - Variation de l'activité massique calculée en fonction de la profondeur pour un béton très lourd. Calculated in-depth specific activity profiles in very heavy concrete. 
${ }^{44} \mathrm{Ca}(\mathrm{n}, \gamma){ }^{45} \mathrm{Ca} ;{ }^{54} \mathrm{Fe}(\mathrm{n}, \gamma){ }^{55} \mathrm{Fe} ;{ }^{151} \mathrm{Eu}(\mathrm{n}, \gamma){ }^{152} \mathrm{Eu} ;{ }^{59} \mathrm{Co}(\mathrm{n}, \gamma){ }^{60} \mathrm{Co}$. Certains radionucléides sont produits par spallation ou par des neutrons de plus de $20 \mathrm{MeV}$. C'est le cas du ${ }^{3} \mathrm{H}$, dû à l'évaporation lors d'une spallation, du ${ }^{22} \mathrm{Na}$, (réaction ${ }^{27} \mathrm{Al}(\mathrm{n}, 2 \mathrm{p} 4 \mathrm{n}){ }^{22} \mathrm{Na}$, l'aluminium étant présent en grande quantité dans le béton), $\mathrm{du}{ }^{54} \mathrm{Mn}$ réactions à seuils de type ${ }^{54} \mathrm{Fe}(\mathrm{n}, \mathrm{p}){ }^{54} \mathrm{Mn}$ et du ${ }^{37} \mathrm{Ar}$. Le tableau III donne, pour les quatre premiers centimètres de béton ordinaire la contribution en activité massique des isotopes produits avec des neutrons de plus de $20 \mathrm{MeV}$. Ces radionucléides représentent $22 \%$ de l'activité massique totale (hors radionucléides naturels).

À la surface des blocs, l'activité totale est plus forte pour du béton très lourd $\left(13 \mathrm{~Bq} \mathrm{~g}^{-1}\right)$ que pour du béton ordinaire $\left(2,4 \mathrm{~Bq} \mathrm{~g}^{-1}\right)$. Ceci est dû à la présence de fer et de Baryum dans les bétons très lourds favorisant d'une part les réactions ${ }^{54} \mathrm{Fe}(\mathrm{n}, \gamma){ }^{55} \mathrm{Fe} ;{ }^{54} \mathrm{Fe}(\mathrm{n}, \mathrm{p}){ }^{54} \mathrm{Mn} ;{ }^{59} \mathrm{Co}(\mathrm{n}, \gamma){ }^{60} \mathrm{Co}$ et d'autre part la réaction ${ }^{132} \mathrm{Ba}(\mathrm{n}, \gamma){ }^{133} \mathrm{Ba}$.

TABLEAU III

Contribution en activité massique des radionucléides produits par des neutrons de plus de $20 \mathrm{MeV}$.

Nucleide specific activity produced by neutrons with energy higher than $20 \mathrm{MeV}$.

$\left.\begin{array}{|lcc|}\hline \text { Radionucléides } & \begin{array}{c}\text { Activité massique }\left(\mathrm{Bq} \mathrm{g}^{-1}\right) \\ \text { toutes réactions confondues }\end{array} & \begin{array}{c}\text { Activite }\left(\mathrm{Bq} \mathrm{g} \mathrm{g}^{-1}\right) \text { produit par } \\ \text { les neutrons de plus de } 20 \mathrm{MeV}\end{array} \\ \hline{ }^{45} \mathrm{Ca} & 8,57 \times 10^{-1} & 3 \times 10^{-3}\end{array}\right]$


L'activité massique en fonction de la profondeur décroît plus vite pour du béton très lourd que pour le béton ordinaire. Le rapport entre l'activité massique à la surface du bloc et à $100 \mathrm{~cm}$ est de 50 pour le béton très lourd et 7,5 pour le béton ordinaire. Ceci est dû à la meilleure atténuation des neutrons dans le béton très lourd que dans le béton léger. Il est à noter que si l'on calcule la longueur d'atténuation $\lambda$ avec :

$$
\begin{aligned}
& \text { Activité à l'entrée } \\
& \text { Activité à } d
\end{aligned}=\exp \left(-\frac{d}{\lambda}\right)
$$

On obtient pour le béton très lourd $\lambda=122 \mathrm{~g} \mathrm{~cm}^{-2}$ et pour le béton ordinaire $\lambda=116 \mathrm{~g} \mathrm{~cm}^{-2}$. Ces valeurs sont à rapprocher de la longueur d'atténuation (en équivalent de dose) couramment utilisée pour des accélérateurs de protons de plus de $1 \mathrm{GeV}$ soit $117 \mathrm{~g} \mathrm{~cm}^{-2}$ pour le béton, le lecteur pourra se référer à Thomas et Stevenson (1988) ou à Bourgois et al. (1996).

\section{Mesures}

\subsection{Matériel et méthodes (Masson, 1999b)}

\subsubsection{Techniques d'échantillonnages}

Pour prélever nos échantillons, on a utilisé un système de carottage au diamant d'une puissance de 1700 watts équipé d'une couronne de forage. Son diamètre externe est compris entre 42 et 62 millimètres, sa longueur est égale à 430 millimètres. Un circuit d'eau de refroidissement composé d'un réservoir sous pression de 10 litres, d'un collecteur et d'un bidon de réception permet de travailler en circuit fermé. Cette récupération des eaux évite tout problème de contamination.

Les carottes de bétons prélevées mesurent en moyenne 360 millimètres de longueur pour un diamètre de 37 millimètres.

Ces carottes sont découpées en tronçons de 40 millimètres grâce à une scie en diamant. Ces échantillons sont broyés, de manière à les rendre le plus homogène possible. La granulométrie obtenue est de 200 micromètres. Ces broyats sont placés dans des flacons de $50 \mathrm{~cm}^{3}$, leurs masses varient entre 70 et 120 grammes selon la densité du béton prélevé.

Pour les bétons très lourds de masse volumique égale à $4,8 \mathrm{~g} \mathrm{~cm}^{-3}$, un conditionnement particulier est effectué pour homogénéiser les échantillons. Celui-ci consiste à séparer le fer du béton par champ magnétique et attaque chimique. 


\subsubsection{Techniques de mesures}

\section{Spectrométrie gamma (Masson, 1999b)}

Pour orienter nos recherches des radionucléides susceptibles d'être présents dans les différents types de béton, nous nous sommes servis des calculs présentés dans ce travail.

Les échantillons de béton sont analysés en spectrométrie gamma par le laboratoire d'analyses nucléaires (L.A.N.) du SPR/CEA-Saclay. L'analyse est effectuée dans une salle bas bruit de fond donnant une limite de détection de $2 \times 10^{-3} \mathrm{~Bq} \mathrm{~g}^{-1}$, pour un temps d'acquisition de 4 heures. Le détecteur au germanium-lithium possède un volume de $100 \mathrm{~cm}^{3}$, son efficacité est de $18 \%$ et il a une résolution de $1,9 \mathrm{keV}$ à $1,33 \mathrm{MeV}$.

Tous les résultats présentés sont corrigés pour tenir compte de l'autoatténuation des échantillons. Les activités mesurées sont recalculées pour un an de décroissance.

\section{Analyse du tritium (Masson, 1999b)}

Le tritium, calculé dans la première partie, est un radionucléide émetteur $\beta^{-}$pur de faible énergie - énergie maximale 18,62 keV (DAMRI, 1991). Pour l'analyser il faut utiliser une technique de scintillation liquide.

Pour chaque tronçon de carotte à examiner, 6 échantillons de 0,1 gramme de béton broyé sont disposés dans des dés en papier. Les trois premiers contiennent du béton seul, dans les trois autres on a ajouté une solution étalon de tritium. Chaque échantillon est disposé dans un appareil oxydiseur de type Sample Model 307 de Packard Canberra Company. Il permet par combustion sous atmosphère enrichie en oxygène, d'extraire le tritium. Ce dernier alors sous forme de vapeur d'eau, peut être piégé et mis en solution par de l'eau distillée. Le liquide scintillant est ajouté automatiquement dans les flacons de $20 \mathrm{~cm}^{3}$.

Tous les résultats présentés sont corrigés pour tenir compte de l'émission de photons lumineux due à l'absorption du rayonnement et aux phénomènes de fluorescence et de scintillation en milieu liquide. Les limites de détections ont varié de 2 à $0,3 \mathrm{~Bq} \mathrm{~g}^{-1}$ suivant le temps d'acquisition.

\section{Analyse du ${ }^{55} \mathrm{Fe}$ (Deslias et Chotard, 1998)}

Le ${ }^{55} \mathrm{Fe}$ est un radionucléide émetteur de rayonnement X de $6 \mathrm{keV}$ (DAMRI, 1991). Cette faible énergie implique des difficultés de détection. Pour la mesure du ${ }^{55} \mathrm{Fe}$ l'analyse comporte deux grandes étapes :

1. obtention d'un concentrat de fer à partir de l'échantillon prélevé,

2. détection $d{ }^{55} \mathrm{Fe}$ par scintillation liquide. 
L'échantillon de métal est découpé puis dissous dans de l'acide chlorhydrique et de l'acide nitrique. La concentration en fer, de la solution mère obtenue, est déterminée par spectrophotométrie d'absorption atomique ou par I.C.P.M.S. (inductively coupled plasma mass spectrometry). Un prélèvement est effectué dans notre solution mère et une précipitation du fer sous forme hydroxyde ferrique est réalisée par un excès d'ammoniaque. Après une centrifugation, puis une dissolution, une élution est faite sur une colonne de résine anionique en milieu $\mathrm{HCl}$.

Pour doser le fer résiduel nous avons réutilisé les mêmes méthodes que pour la solution mère. Connaissant la concentration du départ, on peut en déduire le rendement total de séparation chimique.

Le ${ }^{55} \mathrm{Fe}$ est détecté ensuite par scintillation liquide, le rayonnement $\mathrm{X}$ excite les molécules fluorescentes du scintillant. Le rendement de détection est déterminé avec une solution étalon.

\section{Analyse $\mathrm{du}^{45} \mathrm{Ca}$}

Le ${ }^{45} \mathrm{Ca}$ est un radionucléide émetteur $\beta^{-}$pur d'énergie maximale $256,9 \mathrm{keV}$ (DAMRI, 1991).

Pour effectuer cette analyse nous nous sommes servis d'un mode opératoire décrit par Borak et Awschalom (1972) lors d'une étude portant sur la détection des radionucléides présents dans le sol, auprès d'un accélérateur de protons de haute énergie («National accelerator laboratory» de l'Illinois à Batavia).

La détermination de l'activité $\mathrm{du}{ }^{45} \mathrm{Ca}$ se déroule en deux étapes :

1. Séparation du calcium des autres éléments, en minéralisant un échantillon de 4 grammes de béton. On l'expose à chaud à différentes phases de dissolution, en milieux acides sulfurique, nitrique, fluorhydrique, et chlorhydrique. La solution obtenue est alors évaporée à chaud et reprise en milieu acide nitrique. Ensuite, une précipitation du calcium, sous la forme de carbonate de calcium, est effectuée par ajout de carbonate de sodium. Le rendement de séparation chimique du calcium est déterminé de la même façon que pour le fer, une spectrophotométrie d'absorption atomique est effectuée avant et après la séparation.

2. Scintillation liquide, le rendement de détection est déterminé par le comptage d'une solution étalon.

\subsection{Résultats}

\subsubsection{Béton ordinaire (Masson, 1999b)}

Le résultat de l'activation massique en profondeur pour les émetteurs $\gamma$ dans le béton ordinaire est donné sur la figure 6 . Cette mesure est représentative du bloc de béton 


\section{Activité en Bq.g $\mathrm{g}^{-1}$}

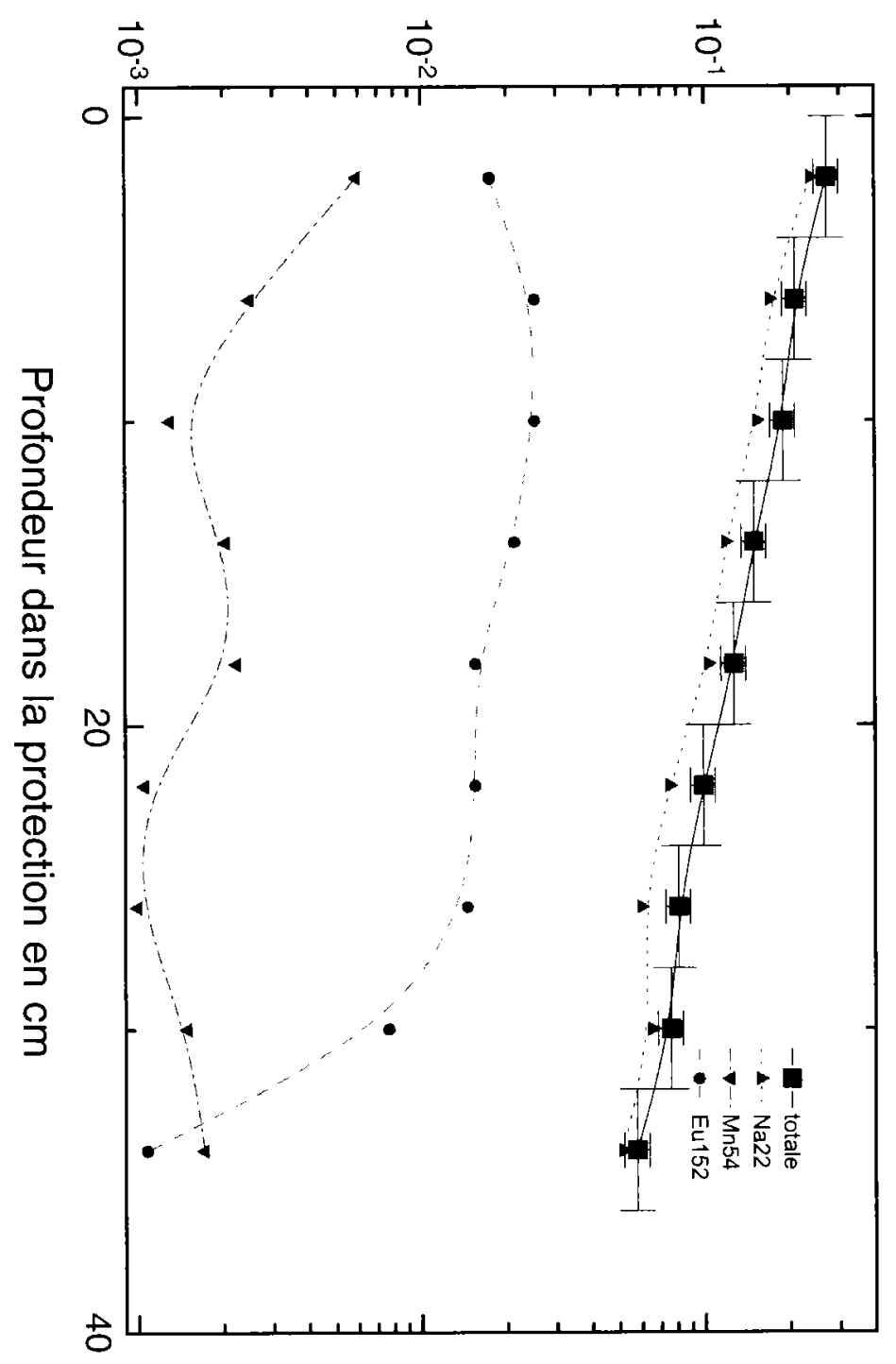

Fig. 6. - Variation de l'activité massique des émetteurs $\gamma$ mesurée en fonction de la profondeur pour un béton ordinaire.

Measured $\gamma$ emetted in-depth specific activity profiles in ordinary concrete. 
ordinaire le plus activé de l'installation. L'activité massique moyenne pour les émetteurs $\gamma$ à l'entrée du bloc est de $0,27 \mathrm{~Bq} \mathrm{~g}^{-1}$, à $34 \mathrm{~cm}$ dans le bloc elle est de $0,06 \mathrm{~Bq} \mathrm{~g}^{-1}$. Cette activation est due en majorité au ${ }^{22} \mathrm{Na}$ créé par spallation sur l'aluminium présent dans le béton.

La valeur de l'activité massique moyenne en tritium obtenue dans les quatre premiers centimètres des prélèvements est égale à $1,5 \pm 0,5 \mathrm{~Bq}^{-1}$ c'est-à-dire très proche de la limite de détection des appareils, soit $1,3 \mathrm{~Bq} \mathrm{~g}^{-1}$.

La valeur de l'activité massique moyenne en ${ }^{45} \mathrm{Ca}$ obtenue dans les quatre premiers centimètres des prélèvements est égale à la limite de détection des appareils, soit $8,5 \mathrm{~Bq}^{-1}$.

\subsubsection{Béton lourd baryté densité 3,6 (Bourgois et al., 1999)}

Le résultat de l'activation massique en profondeur pour les émetteurs $\mathrm{g}$ dans le béton lourd baryté est donné sur la figure 7. Cette mesure est représentative du bloc de béton lourd baryté le plus activé de l'installation. L'activité massique moyenne pour les émetteurs g à l'entrée du bloc est de $4,4 \mathrm{~Bq} \mathrm{~g}^{-1}$, à $100 \mathrm{~cm}$ dans le bloc elle est de $0,038 \mathrm{~Bq} \mathrm{~g} \mathrm{~g}^{-1}$. Cette activation est due en grande partie au ${ }^{133} \mathrm{Ba}$.

La valeur de l'activité massique moyenne en tritium en profondeur est donnée en figure 8. L'activité dans les quatre premiers centimètres est de $2 \mathrm{~Bq} \mathrm{~g}^{-1}$ pour atteindre la limite de détection des appareils $\left(0,3 \mathrm{~Bq} \mathrm{~g}^{-1}\right)$ après $20 \mathrm{~cm}$ de béton.

\subsubsection{Béton très lourd riblonné densité 4,8 (Masson, 1999b)}

Le résultat de l'activation massique en profondeur pour les émetteurs $g$ pour le béton très lourd riblonné est donné sur la figure 9. Cette mesure est représentative du bloc de béton très lourd riblonné le plus activé de l'installation. L'activité massique moyenne pour les émetteurs $\gamma$ à l'entrée du bloc est de $1,1 \mathrm{~Bq} \mathrm{~g}^{-1}$, à $34 \mathrm{~cm}$ dans le bloc elle est de $0,2 \mathrm{~Bq} \mathrm{~g}^{-1}$. Elle est due en majorité aux ${ }^{54} \mathrm{Mn},{ }^{60} \mathrm{Co},{ }^{133} \mathrm{Ba}$.

La valeur de l'activité massique moyenne en tritium obtenue dans les quatre premiers centimètres des prélèvements est égale à la limite de détection des appareils soit $1,9 \mathrm{~Bq} \mathrm{~g}^{-1}$.

La valeur de l'activité massique moyenne en ${ }^{55} \mathrm{Fe}$ obtenue dans les quatre premiers centimètres des prélèvements est égale $6,8 \pm 2,0 \mathrm{~Bq} \mathrm{~g}^{-1}$. L'activité massique moyenne en ${ }^{55} \mathrm{Fe}$ à la profondeur comprise entre 24 et $28 \mathrm{~cm}$ est égale à $4,4 \pm 1,5 \mathrm{~Bq} \mathrm{~g}^{-1}$. 
Activité en Bq.g-1

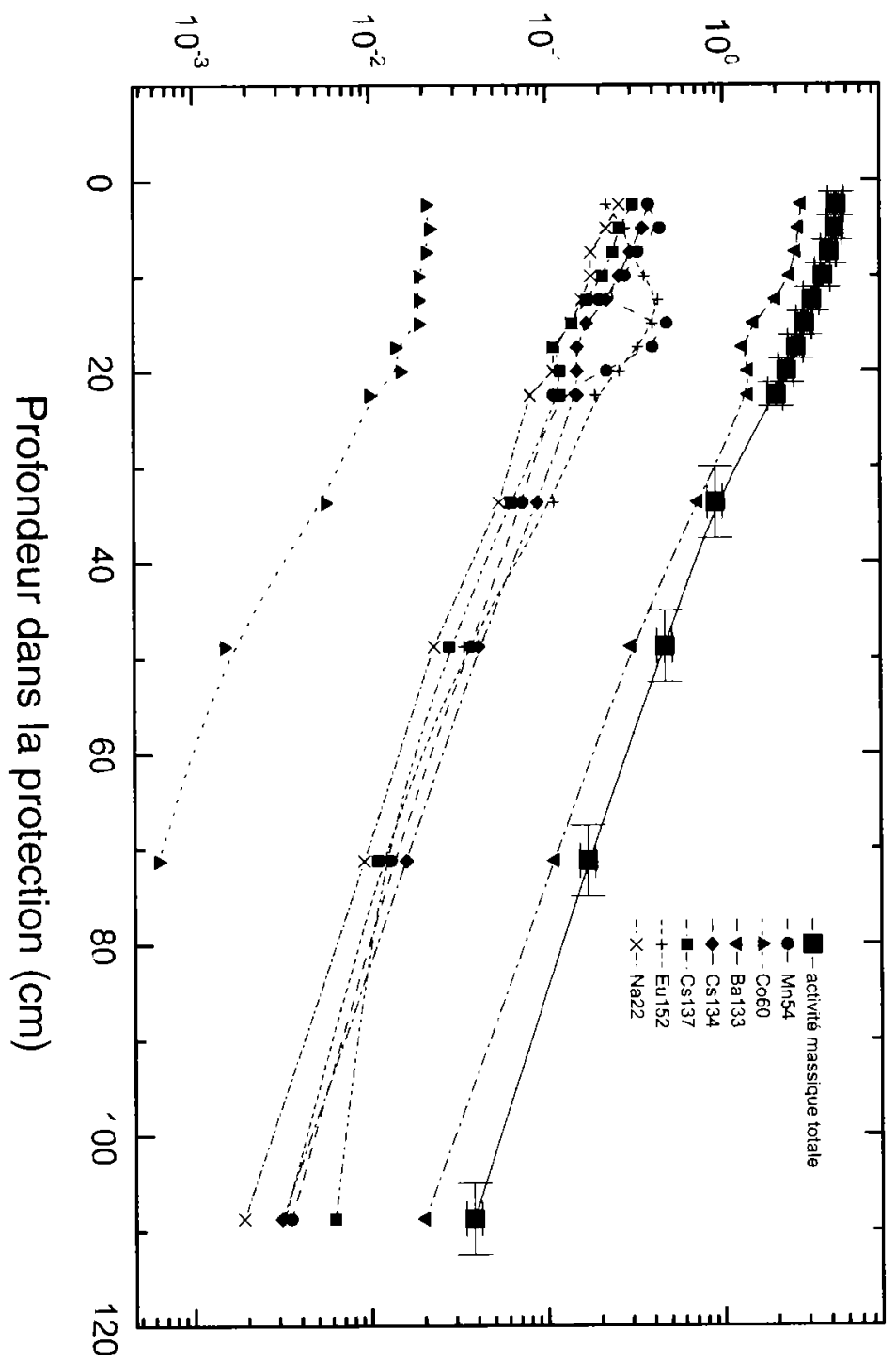

Fig. 7. - Variation de l'activité massique des émetteurs $\gamma$ mesurée en fonction de la profondeur pour un bêton lourd baryté.

Measured $\gamma$ emitted in-depth specific activity profiles in heavy concrete. 


\section{Activité massique en tritium en Bq.g-1}

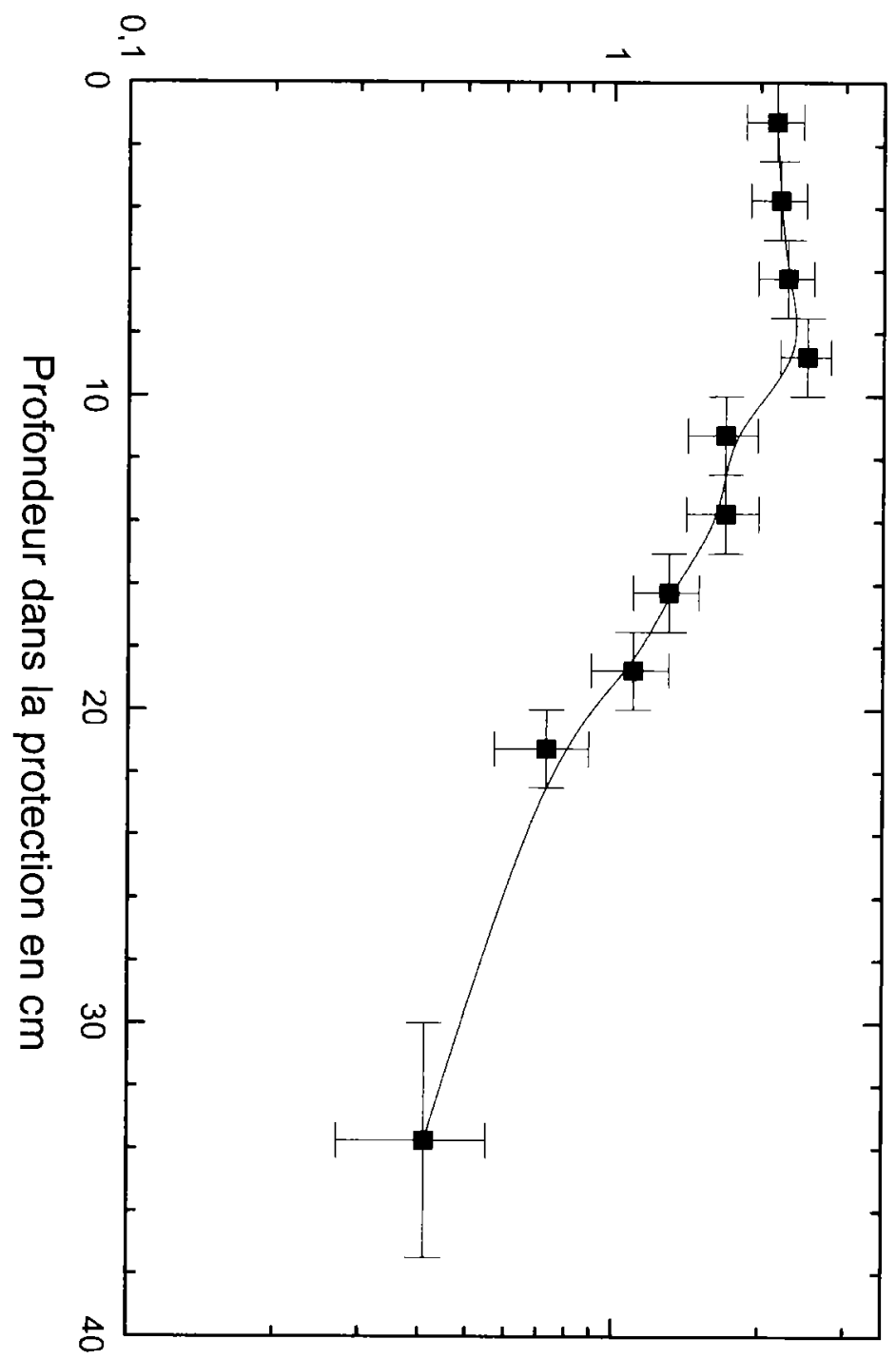

Fig. 8. - Variation de l'activité massique du ${ }^{3} \mathrm{H}$ mesurée en fonction de la profondeur pour un béton lourd baryté.

Measured ${ }^{3} \mathrm{H}$ emitted in-depth specific activity profiles in heavy concrete. 


\section{Activité en Bq. $\mathrm{g}^{-1}$}

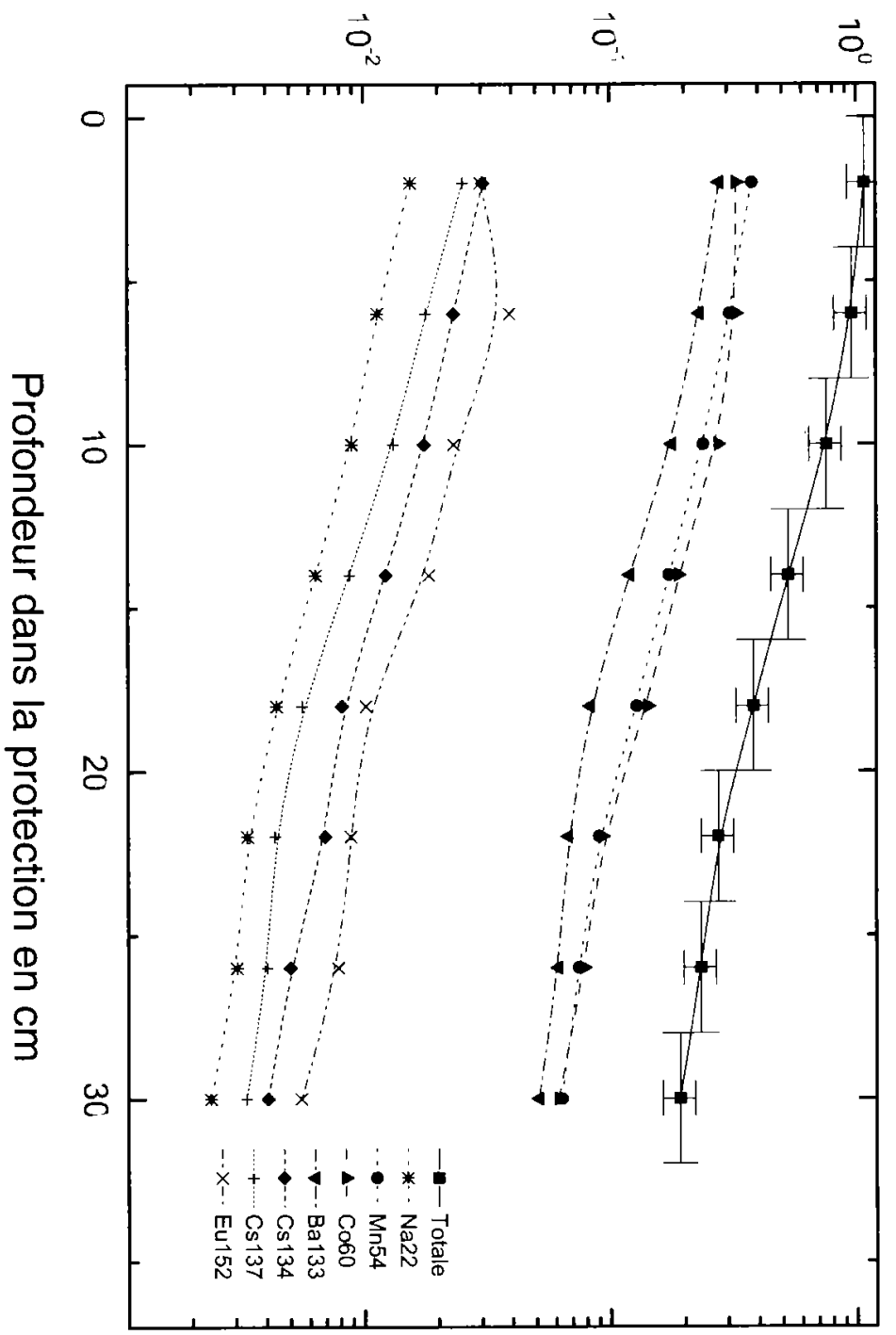

Fig. 9. - Variation de l'activité massique des émetteurs $\gamma$ mesurée en fonction de la profondeur pour un béton lourd riblonné.

Measured $\gamma$ emitted in-depth specific activity profiles in extra heavy concrete. 


\section{Discussion}

\subsection{Comparaison calcul-mesure}

Les figures 10 et 11 donnent pour les émetteurs $\gamma$, le rapport entre les activités massiques théorique et mesurée respectivement dans les bétons ordinaires et très lourds riblonnés.

Pour le béton ordinaire, l'activité mesurée des émetteurs $\gamma$ est proche de l'activité théorique. Le rapport $\mathrm{A}_{\text {théorique }}$ sur $\mathrm{A}_{\text {mesurée }}$ est croissant en fonction de la profondeur et varie de 0,87 pour une profondeur de $2 \mathrm{~cm}$ à 2 pour une profondeur de $35 \mathrm{~cm}$. Le calcul surestime certains radionucléides tels que $1^{152} \mathrm{Eu}$ et le ${ }^{54} \mathrm{Mn}$ alors qu'il en sous-estime d'autre comme le ${ }^{22} \mathrm{Na}$ et le ${ }^{3} \mathrm{H}$. Par exemple, l'activité massique théorique du ${ }^{3} \mathrm{H}$ est sous-estimée d'un facteur 2,5 par rapport à la mesure dans les quatre premiers centimètres.

Pour le béton très lourd riblonné, l'activité mesurée des émetteurs $\gamma$ reste proche de l'activité théorique. Le rapport $A_{\text {théorique }}$ sur $A_{\text {mesuré }}$ est croissant en fonction de la profondeur et varie de 3 pour une profondeur de $2 \mathrm{~cm}$ à 6 pour une profondeur de $30 \mathrm{~cm}$. Le calcul surestime les activités de tous les radionucléides émetteurs $\gamma$. Pour le ${ }^{55} \mathrm{Fe}$, l'activité massique est surestimée d'un facteur 1,4 par rapport à la mesure dans les quatre premiers centimètres, elle est encore surestimée d'un facteur 1,2 à une profondeur comprise entre 24 et $28 \mathrm{~cm}$.

Ces comparaisons montrent toutefois que les estimations théorique et pratique restent du même ordre de grandeur. Les différences entre le calcul et les mesures peuvent provenir de plusieurs facteurs :

- historique de fonctionnement différent (notamment en ce qui concerne les énergies et le type de particules);

- position des mesures différentes de celles calculées;

- géométrie de calcul simplifiée...

\subsection{Comparaison avec d'autres accélérateurs}

Nos résultats mesurés dans le béton ordinaire et à $90^{\circ}$ de la cible ont été comparés à des mesures effectuées sur des accélérateurs différents mais avec des géométries d'irradiation comparables (protection en béton ordinaire à $90^{\circ}$ de la cible). Il s'agissait, d'une part d'un accélérateur d'électrons de $200 \mathrm{MeV}$ de l'IRMM en Belgique et d'autre part d'un cyclotron de production de radioisotopes de $43 \mathrm{MeV}$ en protons de l'université de Bruxelles (VUB). Ces données ont été prises dans le rapport européen sur le démantèlement des accélérateurs (Vankerckoven et al., 1999).

La figure 12 donne l'activité massique en fonction de la profondeur pour ces différents accélérateurs. L'activité massique a été ramenée à une charge intégrée 


\section{Activité théorique / activité mesurée}

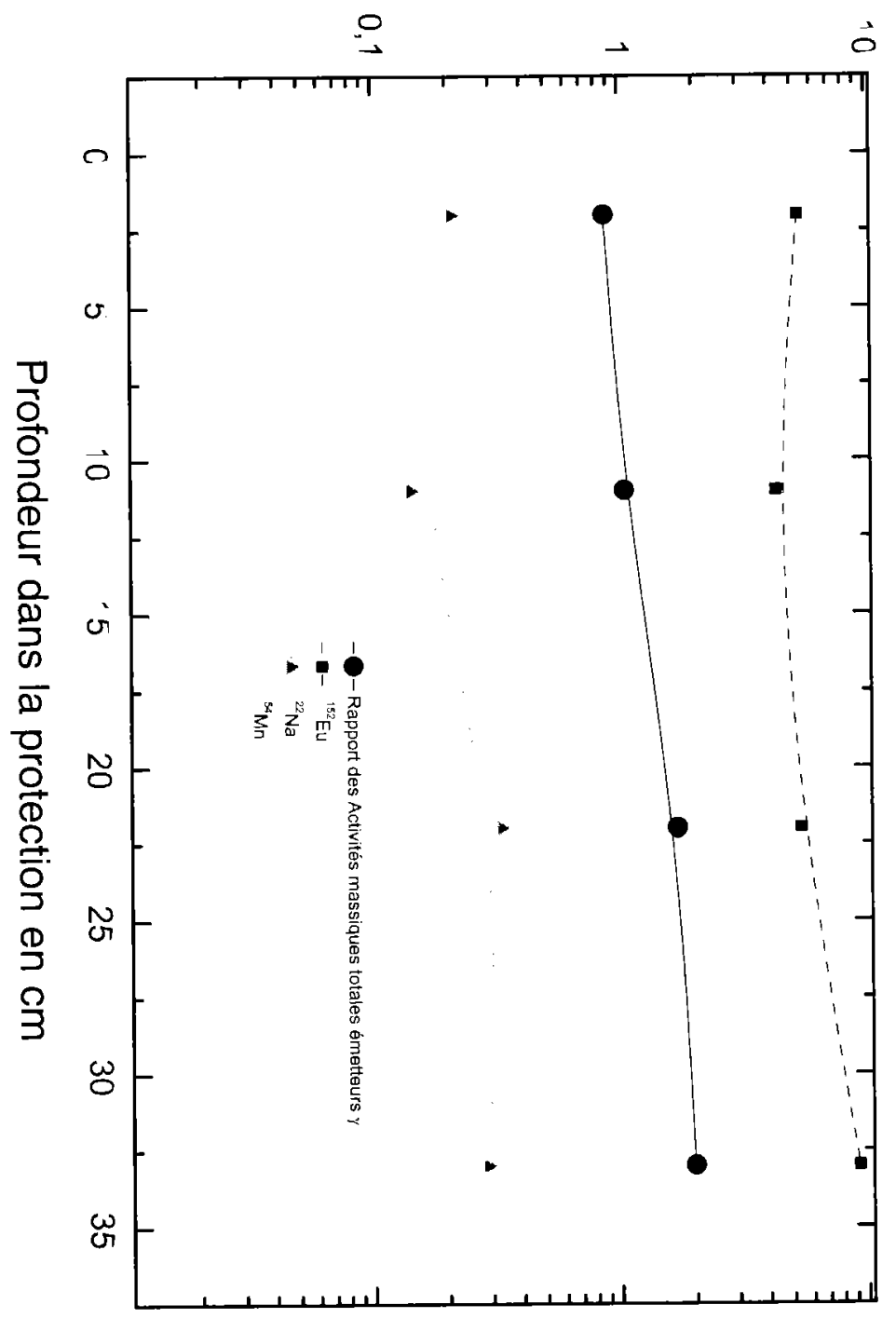

Fig. 10. - Rapport des activités massiques théorique et mesurée pour les émetteurs $\gamma$ en fonction de la profondeur pour un béton ordinaire.

In-depth profile (ordinary concrete) ratio between theoretical results and $\gamma$-ray specific activity measurements. 


\section{Activités massiques théorique / mesurée}

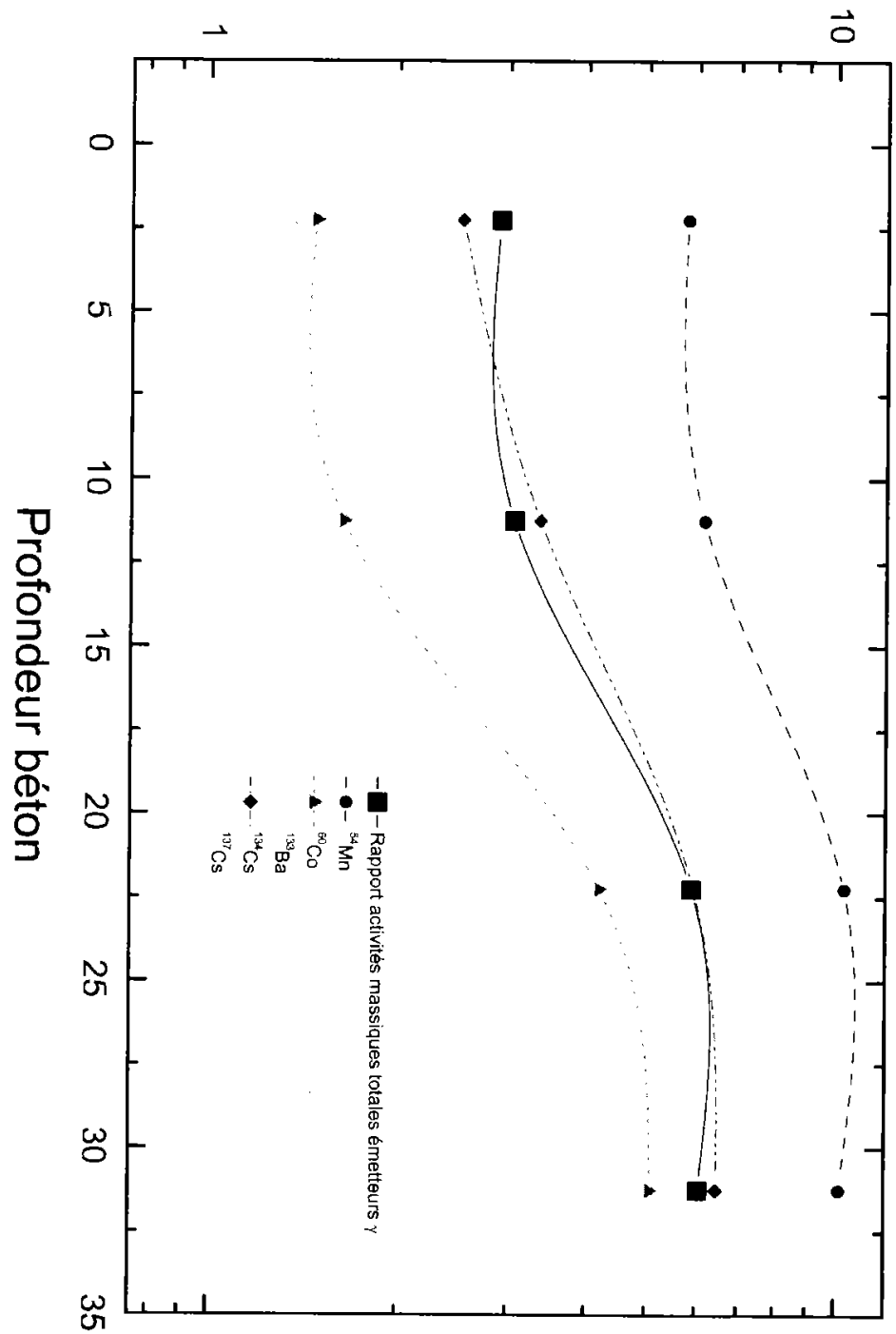

Fig. 11. - Rapport des activités massiques théorique et mesurée pour les émetteurs yen fonction de la profondeur pour un béton très lourd riblonné.

In-depth profile (extra heavy concrete) ratio between theoretical results and rray specific activity measurements. 
Bq. $g^{-1} \mathrm{C}^{-1}$

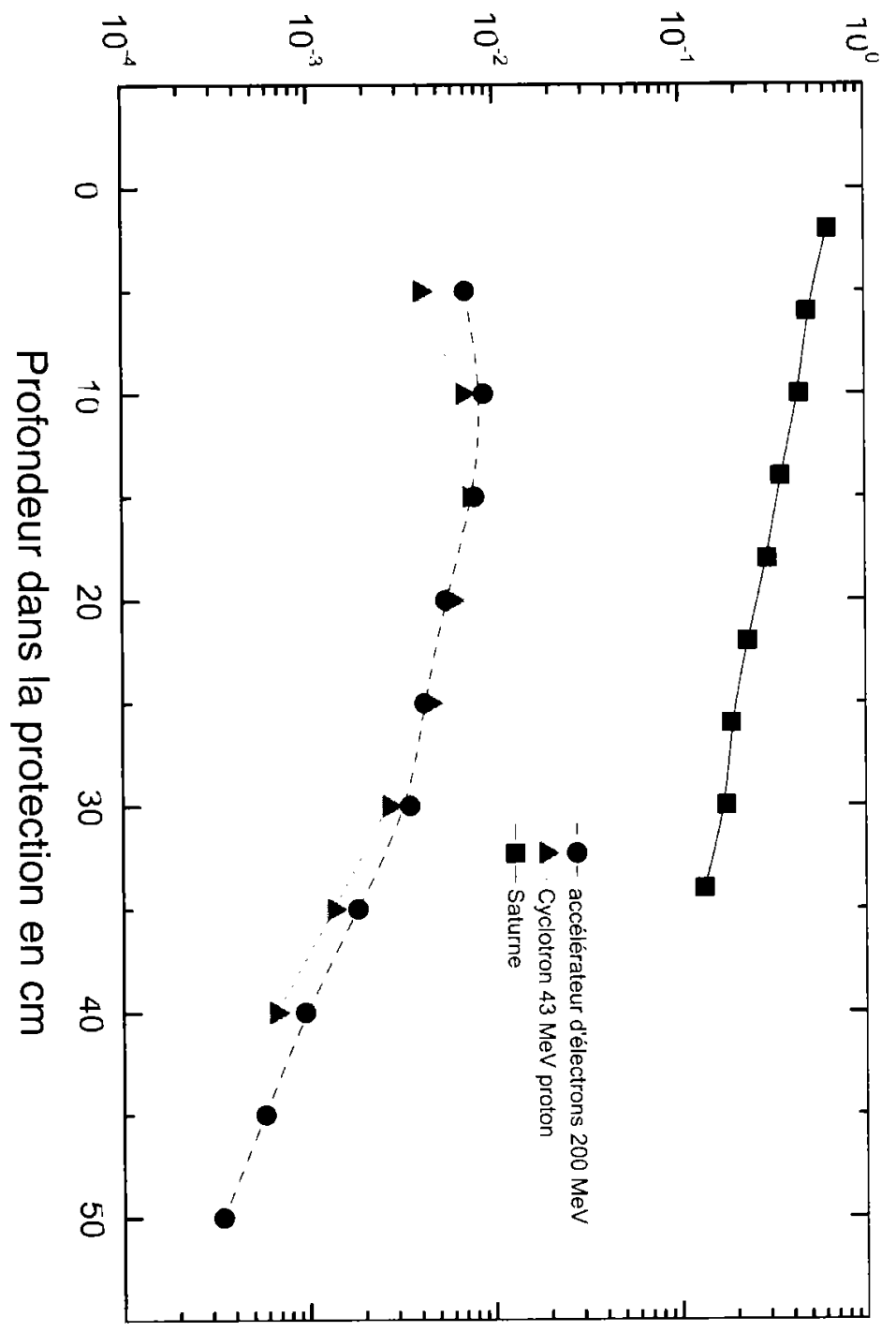

Fig. 12. - Activité massique en profondeur pour différents types d'accélérateur. L'activité massique est ramenée à une charge de $1 \mathrm{C}$ sur la cible.

In-depth specific activity profiles in ordinary concrete for different accelerator for $1 \mathrm{C}$ on the target. 
de 1 coulomb sur la cible (elle était de $1425 \mathrm{C}$ pour le cyclotron, de $8950 \mathrm{C}$ pour l'accélérateur d'électrons et de $0,5 \mathrm{C}$ pour Saturne).

On note que l'activité massique pour $1 \mathrm{C}$ intégré est plus grande dans le cas de l'accélérateur Saturne que dans les autres cas. Ceci est dû au fait que le nombre de neutrons produits pour un coulomb est d'environ :

- 1,25 x $10^{19} \mathrm{n} \mathrm{C}^{-1}$ pour Saturne (Thomas et Stevenson, 1988);

- $7,8 \times 10^{17} \mathrm{n} \mathrm{C}^{-1}$ pour l'accélérateur d'électrons (Swanson, 1979);

- 1,87 x $10^{17} \mathrm{n} \mathrm{C}^{-1}$ pour le cyclotron (Thomas et Stevenson, 1988).

À intensité égale, un accélérateur type Saturne produit 16 fois plus de neutrons qu'un accélérateur d'électrons de $200 \mathrm{MeV}$ et 67 fois plus qu'un cyclotron de protons de $43 \mathrm{MeV}$. On observe que l'activation dans les premiers centimètres de béton pour Saturne est 90 fois plus élevée que pour l'accélérateur d'électrons et 150 fois plus que pour le cyclotron. Les rapports relatifs entre le nombre de neutrons et l'activation varient d'un facteur 2 (cas du cyclotron) à 5 (cas de l'accélérateur d'électrons). Ceci est dû au fait que les temps d'irradiation et de décroissance ainsi que l'énergie des neutrons ne sont pas comparables.

On observe une remontée de l'activité à $10 \mathrm{~cm}$ de profondeur pour l'accélérateur d'électrons et à $15 \mathrm{~cm}$ pour le cyclotron. Celle-ci est due à l' ${ }^{152} \mathrm{Eu}$ (représentant l'essentiel de l'activité dans ces deux cas). Cette remontée est sans doute due à la thermalisation des neutrons dans les protections. Cette remontée n'est pas observée dans les protections de Saturne.

L'activité en fonction de la profondeur décroît plus vite pour les accélérateurs d'électrons et le cyclotron que pour Saturne. Ceci vient du fait que les neutrons pénétrant dans la protection sont moins énergétiques pour ces deux accélérateurs que pour Saturne et sont donc plus fortement atténués.

\section{Conclusion}

Il est possible de prédire l'activité dans les protections d'un accélérateur en utilisant des codes Monte Carlo couplés à un code de calcul d'activation, de filiation et de décroissance. Les activités des émetteurs g calculées semblent surestimées, d'un facteur 2 pour les bétons ordinaires et 6 pour des bétons très lourds riblonnés, par rapport aux activités des émetteurs $\gamma$ réellement mesurées, ce qui va dans le sens du principe de précaution.

On constate un bon accord entre la théorie et la mesure pour le ${ }^{55} \mathrm{Fe}$ et le ${ }^{3} \mathrm{H}$. 
Pour obtenir une bonne adéquation calculs-mesures, le plus difficile est de bien maîtriser les paramètres d'entrée du calcul. Il faut connaître :

- l'historique d'irradiation en terme de particules d'énergie et d'intensité;

- la géométrie d'irradiation;

- la composition précise des protections radiologiques, impuretés comprises.

L'intérêt des calculs est de pouvoir estimer l'activation :

- dans n'importe quel endroit inaccessible de l'installation;

- de radionucléides difficilement mesurables (e.g. émetteurs $\beta$ purs).

Le désavantage est le temps consacré à la préparation des données, à la modélisation, aux calculs et aux dépouillements des résultats.

L'avantage des mesures est le peu de temps à consacrer pour obtenir les premiers résultats. Leur limitation est l'impossibilité de les effectuer n'importe où, de ne pas mesurer certains éléments...

Il est bien évident qu'il serait impossible de carotter l'ensemble des murs de l'accélérateur Saturne - 33000 tonnes de béton (Masson, 1999a). De même il serait illusoire de vouloir simuler tous les recoins de l'accélérateur! Une bonne démarche est de rechercher les points de perte les plus représentatifs, d'effectuer un calcul précis puis de la corroborer par des mesures. Ensuite pour d'autres parties de l'accélérateur, on peut utiliser les calculs et/ou les mesures effectués précédemment en les pondérant par des facteurs pour tenir compte par exemple de taux d'irradiation et de distance différents.

La méthode est applicable à tous types d'accélérateurs. Cependant il convient de remarquer que chaque installation engendre des activations différentes suivant son type de particules accélérées les intensités et les énergies. Il convient de noter que l'énergie de la particule primaire joue un rôle prépondérant sur les valeurs d'activité et sur le profil d'activation en profondeur.

\section{RÉFÉRENCES}

Borak T.B., Awschalom M. (1972) The underground migration of radionuclides produced in soil near high energy proton accelerators, Health Phys. 23, 679-687.

Bourgois L., Delacroix D., Ferreux L., Masson L. (1996) Dose equivalent measurements at a $2.7 \mathrm{GeV}$ proton accelerator and comparison with the Moyer model, Health Phys. 70 (1), 36-40.

Bourgois L., Delacroix D., Zerbib J.C., Masson L. (1999) The analyses of activated materials of "class 4" SATURNE synchrotron. Internal report UGSP/SPR/SRI-T/98-1443 for contribution of European Commission report on "evaluation of the radiological and economic consequences of decommissionning particle accelerators", EUR 1915! EN.

DAMRI (Département des applications et de la métrologie des rayonnements ionisants) (1991) Radionucléides. Recueil regroupant les données principales relatives aux schémas de désintégration des radionucléides les plus utiles. ISBN 2-9064483-03-6.

Deslias M.C., Chotard P. (1998) Dosage du fer 55 dans un échantillon de fer situé dans un bloc de protection de Saturne. Rapport interne DCEA-S/UGSP/SPR/SRSE/98-031. 


\section{MASSON, L. BOURGOIS}

Fasso A., Ferrari A., Rauft J., Sala P.R. (1994) FLUKA : Performances and applications in the intermediate energy range. In: 8th International Conference on Radiation Shielding, Arlington, Texas, USA.

Gaudry A., Bertho X., Piccot D., Boiziau S. (1997) Concentration élémentaire des bétons provenant du laboratoire national Saturne. Rapport du laboratoire Pierte Süe.

I.R.F-CEA (Institut de recherche fondamentale du commissariat à l'energie atomique), I.N.2P.3-CNRS (Institut de physique nucléaire et de physique des particules du centre national de recherche scientifique) (1987) Brochure du laboratoire national Saturne.

Klein J.C., Diop C. (1999) Inventaire et distribution spatiale des radionucléides dans les protections latérales d'un accélérateur de protons. Service d'études des réacteurs et de mathématiques appliquées, Commissariat à l'énergie atomique de Saclay, SERMA/LEPP/RT/99-2512/A.

Masson L. (1999a) Historique de l'accélérateur Saturne. Rapport interne, UGSP/SPR/SRI-T/99-066.

Masson L. (1999b) Détermination de l'activation des matériaux sur un accélérateur de particules en vue de son démantelement. Mémoire d'ingénieur CNAM en sciences et technologies nucléaires.

Swanson W. (1979) International atomic energy agency radiological safety aspects of the operation of electron linear accelerators. Technical Reports Series $n^{\circ} 188$, Vienna.

Thomas R.H., Stevenson G.R. (1988) Intemational atomic energy agency radiological safety aspects of the operation of proton accelerators. Technical Reports Series $n^{\circ} 283$, Vienna.

Tsilanizara A. et al. (1999) DARWIN : an evolution code system for a large range of applications. Communication to ICRS-9, Tsukuba, Japan, J. Nucl. Sci. Technol. (to appear).

Vankerckoven P., Hermanne A., Eggermont G., Sonck M., Buls N., Mol H., Sćhrauben M., Verstraeten I., Meier F., Salomé J.M., Peeters L., Vansuetendael W., Zerbib J.C., Bourgois L., Delacroix D., Aït H., Malambu E., Bellefontaine C., Vermeersh F., Vandeplassche D., Meert D. (1999) Evaluation of the radiological and economic consequences of decommissionning particle accelerators, EUR $19151 \mathrm{EN}, \mathrm{ISBN} 92-828-7222-\mathrm{X}$. 